\title{
Frontera política y espacio fronterizo
}

\author{
Arturo Ranfla González \\ Instituto de Investigaciones Sociales \\ Universidad Autónoma de Baja California
}

\begin{abstract}
Resumen
La propuesta de este trabajo es que la frontera no es solamente un instrumento de demarcación territorial como lo sugiere su definición formal; no es una entidad neutra, ni un instrumento absoluto de cada Estado según las líneas discretas y frágiles a que las relegan las cartas geográficas. Lo relevante es que la frontera es un fenómeno social. Como tal, da lugar a la presencia de conflictos, discordancias y transformaciones que la ubican en una dimensión dinámica. Esto la lleva a engendrar costos, des-economías y des-funcionalizaciones cuyos efectos inciden directamente sobre las poblaciones fronterizas e indirectamente sobre los complejos nacionales. El espacio fronterizo, como complejo social, posee la originalidad de establecer un punto fijo en el análisis de formas históricas que resultan de la experiencia, percepción y construcción del espacio.
\end{abstract}

Palabras clave: demarcación territorial, discordancias, transformaciones, dimensión dinámica, dominación.

\begin{abstract}
This work proposes that the border is not only an instrument used for territorial delimitations, as it is stated on its formal definition; neither a neutral entity, nor an absolute instrument installed by each state according to the discreet and frail lines of the geographical charts. The outstanding point is that the border is a social phenomenon that results on conflicts, discordance, and changes on itself becoming a dynamic dimension. In this way, the border gives birth to costs, anti-economics, and anti-functionalities whose effects directly affect the border cities and indirectly bear influence on the domestic complexes. As a social complex, the border space has the characteristic to establish a fixed point and analyze the historical forms resulting from the experience, perception, and construction of spaces.
\end{abstract}

Keywords: territorial delimitation, discordances, transformations, dynamic dimension, domination. 


\title{
FRONTERA POLITICA Y ESPACIO FRONTERIZO
}

\author{
Por \\ Arturo Ranfla Gonzâlez \\ Investigador del I.I.S.
}

Espacio y territorio son términos utilizados frecuentemente como sinónimos en la investigación social. La ambigüedad en su utilización es el resultado de la complejidad en que se ubica la categoría espacio; el espacio que a la par del tiempo es categoría fundamental en el desarrollo del logos occidental: ambas poseen un carácter relativo que las convierte en instrumentos privilegiados del conocimiento empírico.

En este universo complejo, espacio y territorio sirven para designar indistintamente soporte físico o espacios abstractos (definidos a priori). En realidad, mientras la categoría espacio posee la doble dimensión por la cual oscila entre la abstracción y la materialidad, el territorio es un concepto preciso, que se define en relación a la sensibilidad exterior que lo identifica por medio de coordenadas (dimensión exclusivamente física). Esta aparente confusión que se deriva en el uso indiscriminado de ambos términos, no constituye más que una forma de excluir la contradicción que se desarrolla por la oposición que puede existir entre la territorialidad de todo poder político y la abstracción de los espacios de organización. ${ }^{1}$

El espacio es la categoría universal en la que se integran los espacios abstractos de organización que se conciben y las estructuras territoriales que se construyen. Organización y construcción del espacio reflejan, por sus estructuras y transformaciones, el dominio de dos criterios centrales; primero, de carácter económico y en el cual se sustenta la toma de decisiones de los agentes individuales y colectivos; el segundo, de carácter político, que sustenta y garantiza el ejercicio de la soberanía inherente a la existencia de todo Estado-nación.

Más allá de toda tendencia de simplificación, el espacio externa su complejidad frente a las necesidades del análisis empirista o la intuición idealista de los espacios abstractos 2. El espacio como categoría universal va a escapar sistemáticamente a todo reduccionismo, al emerger como producto complejo

\footnotetext{
1 Claval P., Espace et pouvoir, PUF, Paris, 1978, Gottmann J., The significance of territory, Charlettosville, The University Press of Virginia, 1983 ; Perroux E, Pouvoir et Economie, Dunod, París, 1974.

2 La interpretación de Kant respecto al espacio como "forma de intuición" o de "intuición pura", permitió a la geometrfa garantizar su papel de ciencia que determina las propiedades del espacio a priori, para oponerlo a toda experiencia.
} 
de las estructuras territoriales, por los lugares y localizaciones asignadas y las formas simbólicas en las que se desarrollan, coexisten y organizan las fuerzas productivas.

El espacio, al incorporar los actos sociales de sujetos individuales y colectivos, es un producto del desarrollo histórico de las relaciones de producción y poder. El espacio social, como proceso temporal, va a integrar las formas de organización históricamente determinadas por cada sociedad en relación a sus localizaciones privilegiadas. Territorialmente, la distribución de población y edificios (formas construidas) va a asegurar la cohesión necesaria en el desarrollo del proceso productivo; simbólicamente, edificios, monumentos y obras de arte van a reflejar el estado de las relaciones de producción y de poder que se desarrollan en cada sociedad.

El espacio social como entidad compleja puede, en aras de su inteligibilidad conceptual, plantearse en base a una triplicidad en la cual convergen dimensión física (territorial), criterios individuales y colectivos de percepción y diferentes formas de representación ${ }^{3}$.

La práctica espacial como proceso que engloba producción y reproducción en relación a la localización geográfica y a los movimientos, emerge como forma espacial original de toda sociedad históricamente determinada. La práctica espacial como dimensión espacio-temporal es el factor que asegura la continuidad del desarrollo social dentro de una cohesión relativa.

La representación del espacio. Ligadas a las diferentes "racionalidades" que se desarrollan en el seno de toda sociedad, las normas que ellas instituyen y a los criterios del espacio a que dan lugar, la representación del espacio es un conjunto de símbolos y códigos dominantes en el cual se desarrollan las relaciones "frontales" de poder (económico y político), de los agentes dominantes de la percepción del espacio social.

Los espacios de representación. Ellos constituyen la simbólica marginal de símbolos y códigos complejos que vehiculan y se constituyen en circuito marginal y clandestino de la vida social. Temporalmente, esto los convierte en códigos potenciales de recambio en la percepción social del espacio.

En esta triplicidad, el espacio es una realidad material que refleja el desarróllo histórico de cada sociedad. El es la resultante de la producción y re-

3 'D autant que l'espace ainsi produit sert aussi d'instrument á la pensée comme á l'action, qu'il est, en même temps qu'un moýen de production, un moyen de contrôle donc de domination et de puissance", Lefebvre H., La production de l'space, Anthropos, París, 1981, p. 35. Esta complejidad y su comprensión nos llevan a utilizar los conceptos del mismo autor en la reflexión del espacio, pp. 46-57 
producción de los comportamientos y códigos dominantes, al mismo tiempo que de la emergencia de prácticas marginales o vedadas como elementos dinámicos. Diacrónicamente, esto da lugar a identificar la etimología del espacio en relación a las modificaciones de lugares y formas. Sincrónicamente, el espacio da fe de una práctica actual y cotidiana que se expresa por sus relaciones y conexiones.

En el contexto dinámico en el que se estructura y construye el espacio, la frontera política como corte territorial-temporal, va a introducir una dimensión estática en la comprensión y análisis del desarrollo histórico y las transformaciones espaciales de toda sociedad. Al constituirse, la frontera política, condición de existencia a todo Estado-nación en relación al territorio, se convierte directa $\mathrm{e}$ indirectamente en un factor de transformación del espacio.

La frontera política, en su doble dimensión jurídica y territorial, interviene como factor de distorsión o amputación en la producción y reproducción de los espacios de organización económica y política. La contigüidad geográfica entre entidades políticas a que remite su propia definición, hace de ella lugar de confrontación de al menos dos procesos de integración política interna, de organización administrativa y en ocasiones de carácter étnico y cultural. El resultado es una realidad dinámica que emerge para integrar estas diferencias originales a otras dimensiones de la organización social.

La entidad emergente que es el espacio fronterizo, es un producto territorial y de formas de organización que resulta de las normas y códigos impuestos por cada uno de los Estados y su respectiva realidad socioeconómica que se ajustan e infringen, en su caso, las fronteras formales. El espacio fronterizo es, en estas condiciones, la constatación del carácter social del espacio que se rebela a toda concepción normativa, que al fijarlo, lo convierte en una realidad neutra.

El espacio fronterizo es una realidad cuya organización puede definirse de una manera general en base a dos instancias; una vertical, de carácter jurídico, y que el derecho se esfuerza por regular de manera precisa; otra horizọtal, que va más allá de toda legalidad y que traduce las tendencias históricas y las diferencias en el desarrollo histórico de las sociedades nacionales que comparten la misma frontera internacional. En este contexto, su originalidad consiste en emerger como producto territorial de un doble proceso espacial:

- Por un lado, aquel que emana de la estabilidad y el ejercicio de una autoridad central (política), que al instrumentar e implementar me- 
canismos de control y vigilancia, tiende a estructurar un conjunto de relaciones centrípetas orientadas a partir de la línea fronteriza.

- Por otro lado, aquel que corresponde al desarrollo y al movimiento de fuerzas socioeconómicas que, rebasando el ámbito de control de las autoridades centrales, se manifiesta como un conjunto de fuerzas centrífugas que se desplazan en relación a la frontera política.

El espacio fronterizo es una realidad dinámica que,en oposición al criterio generalizado que la convierte en punto de expiración de la realidad socioeconómica de cada país, tiende como fenómeno regional a subrayar su carácter no neutro e interactivo. La región fronteriza como entidad territorial diferenciada desarrolla estructuras de organización que se desarrollan a partir de sus diferencias internas (internacionales) y cuya articulación espacial y temporal sufre transformaciones en función de decisiones y disposiciones que emanan de cada una de las autoridades políticas centrales de cada país.

\section{LA FRONTERA POLITICA}

Todo intento de análisis sobre el papel e impacto de la frontera política en la estructuración y desestructuración del espacio, nos lleva a recurrir en primera instancia a su definición formal. Como institución jurídica ella condiciona su existencia a la presencia de un territorio y un Estado, el que a su vez existe en base a la población y a un territorio dado. En estas condiciones, la frontera es una institución cuya dimensión histórica es la de constituir el punto de equilibrio entre el territorio, la nación y el Estado.

La frontera tiene una existencia material en base al principio de territorialidad que ella posee. Delimitar la jurisdicción territorial es una necesidad de todo Estado-nación en un régimen de estados múltiples, necesidad que aparece como condición previa a su estabilización y consolidación. Como necesidad política, ella es una delimitación territorial continua y estable, que conduce a la noción generalizada de la frontera línea.

La distribución que los Estados modernos hacen de sus territorios evacúa toda posible interferencia y yuxtaposición. En base al principio de exclusividad territorial las fronteras son rigurosamente delimitadas; en consecuencia, Ia frontera línea y su demarcación son una regla en el seno del derecho internacional y un recurso previo al espacio de poder soberano de todo Estado-nación.

Históricamente, la delimitación precisa de la frontera obedecra a condiciones socio-políticas y socio-económicas que llevaban a la formación de 
Estados unitarios y centralizados 4 . En la actualidad, para un gran númcro de casos, Ia frontera fija aparece como una abstracción frente a la explosión geográfica en el intercambio de bienes y el movimiento de factores a escala mundial. En este fenómeno generalizado, el espacio fronterizo externa una doble originalidad: catalizar por medio de la contigüidad geográfica la intensidad y el volumen de los movimientos y desarrollar formas de regulación institucional, cuyos efectos inciden en su configuración organizacional y territorial.

Frente a esta constatación, el derecho internacional, a la par de otras disciplinas sociales, plantea la disyuntiva de analizar la frontera dentro de una óptica zonal que desvanezca la parcialidad que posee el enfoque tradicional, el cual va a asimilarla a un corte lineal, absoluto y neutro.

\section{La frontera línea.}

La frontera lineal es la noción más aceptada que existe de las fronteras internacionales. En relación a esta definición general existen dos criterios dominantes: aquel en que la frontera, strictu sensu, marca el punto de expiración de dos soberanías; un segundo, en donde la frontera separa territorios en los cuales el Estado no ejerce más que parcialmente sus competencias 5. En ambos casos, la frontera, al delimitar su soberanía, define al mismo tiempo su espacio de competencia territorial plenaria.

Independientemente de que la competencia territorial expire en un punto fijo (definido en relación a coordenadas geográficas), la frontera permanece como concepto unitario y homogéneo en relación a su carácter voluntarista y objetivo. En el primer caso, la frontera se convierte en una entidad material resultante de acciones políticas y técnicas que permiten a cada país delimitar (territorialmente) su frontera, mientras la objetividad de la frontera es inmanente a la existencia material de cada Estado y su estabilidad. En consecuencia, toda modificación al trazo de la frontera original remite directamente a una confrontación violenta o tratados desiguales entre países vecinos, lo que lleva a su redefinición en el tiempo y el espacio.

\section{La frontera-zona}

La noción de frontera-zona plantea un problema a los juristas, en virtud de promover la oposición al desarrollo de un derecho internacional normativo, para el cual la frontera se define en base a líneas netas, precisas y, en la

+ Marx K., La cuestión nacional y la fomación de los Estados., Siglo XXI Edit., México, 1980; una visión organicista es la de Ratzel F., Geografia Política.

5 Blumman C., "Frontiéres et limites", in Frontiere, Colloque de Poitiers (AJLF), Edit. Pedone, Patis, 1980, p. 8 
medida de lo posible, exactas. Esta oposición se convierte en el punto central del conflicto entre el derecho normativo y el derecho positivo, donde el segundo se esfuerza por integrar la realidad en oposición a las demarcaciones precisas. Con este objeto, el derecho positivo introduce y desarrolla la idea de confines 6 para integrar la dimensión dinámica a la cual responde la realidad fronteriza.

Los confines-extremos. En tanto que extremidad, los confines se sitúan en relación a un régimen de derecho interno, independientemente de sus efectos sobre el derecho internacional y sobre los Fistados vecinos, Las zonas fronterizas disfrutan frecuentemente de estatutos específicos, ya sea administrativamente o sobre el plan político. Administrativamente, la mayor parte de los Fistados delimitan una zona fronteriza en las cuales se aplican regimenes particulares; políticamente, Ios confines conducen a los Estados a decisiones delicadas en cuanto ellas inciden directamente en las tensiones que pueden originarse en estos territorios.

En estas condiciones, cada Fstado debe considerar la especificidad a que están sujetas sus respectivas fronteras. Las decisiones y aplicación de mecanismos de regulación deben remitirse a la dinámica y a la talla que tienen estos espacios marginales en el contexto de sus respectivos Estados nacionales.

Los confines-ligadura. La noción de ligadura tiene una connotación en derecho internacional que remite a cooperación entre Estados vecinos. La noción de confín-ligadura adquiere de esta manera una dimensión internacional. En la práctica, el derecho internacional se rehusa a aceptar esta noción, en virtud de atentar contra el principio normativo de soberanía absoluta de los territorios nacionales. El resultado es que, jurídicamente, el concepto de confín no exista. Mientras, su emergencia en el plano socioeconómico to convierte en una realidad susceptible de ser localizada. Todo esto lleva a constatar el desfase entre el concepto jurídico y la realidad fronteriza, oposición que va a dar lugar a un mecanismo de recuperación en el plano formal, cuyo efecto es el crecimiento de los acuerdos bilaterales en un gran número de territorios fronterizos 7 .

Fista visión dinámica del espacio fronterizo se desarrolla en relación a dos grupos de tendencias seculares, a partir de las cuales se organizan teóricamente las entidades territoriales nacionales.

\footnotetext{
- I.a expresión de confín tiene un doble sentido en relación a las raíces latinas que la forman: "finis" sobreentiende la expiración de un territorio, alejamiento en relación al corazón, a la capital, $\mathrm{t}: 1$ "cum" define, por el contrario, la idea de ligadura con un espacio vecino. Ella evoca una rona de transición y de pasaje, lo que conduce a establecer la diferencia entre confínextremidad y confín-ligadura.

7 Kiss R. "La frontićre de cooperation", in Frontiere, op, cit., p. 181.
} 
- La primera, plantea el desarrollo histórico de todo Estado-nación y su consolidación en base a la integración política de su territorio. $\mathrm{Si}$ guiendo un principio de "equilibrio" político-territorial, todo Estado justifica la aplicación de instrumentos de regulación como medios para garantizar su unicidad. Internamente, todo Estado promueve este proceso reduciendo las fuerzas centrifugas y las tensiones que espacialmente tienen una mayor sensibilidad en los territorios fronterizos.

- La segunda, pone de manifiesto el carácter estable que, se asume, tiene todo Estado-nación. De esta manera, el Estado es una entidad global capaz de organizar y planificar su territorio, convirtiéndose en una dimensión estática que reduce su territorio de jurisdicción a repeticiones y circularidades, al suponer que controla y regula de una manera absoluta el desplazamiento de sus ajustes sociales más allá de sus fronteras.

Lo anterior pone de relieve la existencia de un vacío entre la realidad social y la norma jurídica que se manifiesta en los espacios fronterizos. En este sentido, la noción de confín-ligadura es una aportación necesaria a la comprensión de estos espacios. Al oponerse sistemáticamente a la fijación del tiempo y el espacio, dicha noción hace que la frontera jurídica aparezca como un código constantemente rebasado por el movimiento dialéctico en que se desarrolla el espacio social. En consecuencia, frente a la rigidez propia de la norma jurídica, la recuperación de las nuevas formas de organización en que se desarrolla la frontera lleva a la necesidad de formular una cada vez mayor número de tratados, acuerdos y convenios bilaterales en relación a las fronteras internacionales,

\section{EL ESPACIO FRONTERIZO}

Una vez que se conviene en señalar a la frontera como dimensión zonal, y que la idea de autonomf́a e impermeabilidad absoluta entre territorios nacionales se desvanece, el concepto de movilidad aparece como un recurso que puede facilitar la comprensión de la dinámica fronteriza.

La movilidad como cambio de localización adquiere su definición más convencional a nivel internacional en relación al desplazamiento de la población y de los bienes. Estos movimientos tienen en la frontera la particularidad de organizar el espacio según procesos de articulación o integración, que involucran directamente los factores de carácter socioeconómico y propiamente territoriales, etc. La forma especifica en que se desarrolla cada uno de estos procesos refleja las condiciones estructurales que tiene cada uno de los Estados nacionales y el margen de negociación y/o regulación de que disponen en relación al país vecino. 
En el terreno específico del análisis económico, la apertura en base a la cual se organiza el espacio fronterizo da cuenta de su heterogeneidad en oposición a la teorfa clásica del comercio internacional. El espacio fronterizo tiene la originalidad de aparecer como caja de resonancia en que emergen los efectos de dominación, los procesos acumulativos en los diferenciales de desarrollo y la estructuración y desestructuración de sus formas territoriales.

Espacialmente, la apertura relativa en que se desarrollan las fronteras internacionales es una condición que permite asimilar estos espacios en una óptica regional. El territorio fronterizo va a estructurarse en base a los flujos de bienes, factores, información, etc. Tanto la intensidad como la dirección de tales movimientos tienden a traducir las relaciones de carácter global en que se organizan los países geográficamente contiguos. Así, la región fronteriza es un espacio de organización política y socioeconómica que exhibe los fenómenos que se desarrollan en cada uno de los países, al mismo tiempo que es una forma territorial privilegiada que refleja las diferencias en base a las cuales se estructuran sus relaciones bilaterales.

\section{Articulación e integración.}

La frontera como dimensión espacial es percibida en relación a la articulación de sus estructuras polfticas nacionales, criterio general que traspuesto al análisis económico ortodoxo ha fructificado en la percepción generalizada de un espacio fronterizo neutro. La constatación de relaciones de carácter asimétrico tanto en el plano político como en el económico, apuntan más hacia un proceso de integración en virtud del cual define dirección e intensidad.

Articulación e integración son así, en la dimensión espacial fronteriza, dos procesos que definen individualmente las instancias centrales que estructuran estos espacios: la normativa, que deriva del poder político interno y su dimensión territorial; y la funcional, que obedece a la racionalidad individual y corporativa de carácter socioeconómico. Ambas instancias, no siendo factibles de exclusión, reflejan el carácter complejo del espacio fronterizo al que encubre la persistencia de las retóricas nacionalistas que excluyen todo proceso regional fronterizo.

El análisis económico no escapa a esta tendencia globalizante, para la cual la relación entre dos economías nacionales se limita a una articulación en base al solo movimiento de bienes. Siguiendo la hipótesis central de unidad territorial de la estructura productiva, la existencia de la región fronteriza será excluida. El análisis neoclásico,como ejemplo más acabado de este criterio de unidad estructural-territorial de toda economía nacional, va a recurrir a la hipótesis extrema de inmovilidad del trabajo y del capital para justificar tal separación. 
Espacialmente, la determinación de un sistema jerárquico de localizaciones industriales llevó a los teóricos de los lugares centrales a plantearse el problema de la marginalidad de los espacios fronterizos. Suponiendo una inmovilidad internacional relativa de capital y trabajo, las estructuras de localización que resultan de las áreas de mercado son distorsionadas por la presencia de la frontera política y sus funciones reguladoras. De este modo, el espacio fronterizo es percibido como amputación a las áreas de mercado por la presencia del principio de separación sociopolítica. ${ }^{8}$

$\mathrm{La}$ integración como proceso que describe un proceso dinámico aparece impregnado de cierta ambigüedad. Por un lado, porque subraya el carácter suboptimal de toda integración, salvo el caso límite 9. Por el otro, porque ló define como acto voluntario en base a objetivos de unidad y solidaridad entre entidades e individuos. En el contexto fronterizo, la integración desvanece la neutralidad a que remite la idea de articulación entre territorios nacionales, en virtud de la existencia de mecanismos de ajuste regional e internacional en base a los cuales se organizan estos territorios.

Territorialmente la integración aparece como un caso límite en cuanto se refiere a la frontera, ya que en realidad este límite no posee una dimensión absoluta más que en el plano jurídico. Espacialmente el fenómeno es más complejo, en virtud de que la voluntad de integrar y su dirección poseen una doble dimensión de carácter normativo (político) y funcional (socioeconómica). El primero refleja el estado de las relaciones bilaterales y cuyos efectos se manifiestan indirectamente en la intensidad y dirección de las funciones fronterizas. EI segundo obedece al comportamiento individual y colectivo de los agentes que organizan sus desplazamientos en función de la desigualdad entre las estructuras nacionales y sus relaciones asimétricas.

Localizado en la frontera, el proceso de integración adquiere una doble dimensión con respecto al punto fijo con el que es equiparada la línea fronteriza. Las estructuras territoriales y los espacios de organización (espacio fronterizo) son una manifestación de la voluntad y la capacidad de integración política de los gobiernos centrales a través de sus mecanismos de regulación. Al mismo tiempo, dichas estructuras reproducen territorialmente las diferencias cualitativas y cuantitativas de la organización de los paŕses limítrofes.

A partir de la línea fronteriza, integración y desintegración aparecen como procesos simultáneos en base a los cuales se estructuran estos territorios. En su alternancia, las determinaciones polfticas y económicas ha-

${ }^{8}$ Christaller W., Central places in Soutbem Germany., Prentice Hall, Englewood Cliffs, N.J., 1966, p. $77-80$.

9 Marchal A., L'intégration temitoriale, PUF, París, 1965. p. 10-15 
cen de estas regiones entidades particularmente dinámicas. Esta concepciór descarta la noción ratzeliana para la cual la frontera política se beneficiaba de la estabilidad y permanencia de las organizaciones políticas. Esta transformación encuentra su explicación en la dinámica económica que sistemática. mente rebasa las líneas formales, siguiendo las necesidades de expansión de los modernos espacios de organización económica.

La frontera da lugar a una entidad regional que se estructura a través de movimientos pendulares de población (trabajadores-consumidores), inversiones industriales, adquisiciones inmobiliarias, etc. En esta nueva forma de organización, los problemas de frontera dejan su lugar a los problemas fronterizos. De la confrontación a la que remite tradicionalmente su presencia, esa organización da lugar a mecanismos de regulación de carácter legal, inductivos o de control, de los cuales hace uso cada poder central en relación a los desplazamientos que se desarrollan.

Las funciones de regulación que ejerce la frontera son relativos a los hombres y a los bienes. Su objeto es controlar, vigilar, reducir, prohibir o excluir el movimiento que de ellos se realiza entre territorios nacionales. En el caso de países que comparten territorialmente una misma frontera, el espacio resultante es extremadamente sensible, por conjugar las diferencias socioeconómicas y los mecanismos de regulación que se desarrollan al interior de cada uno de los países involucrados. En consecuencia, la forma que adquieren las funciones fronterizas y su ejercicio van a oscilar entre un criterio formal que va en el sentido de la integridad territorial (soberanra) y un proceso de interdependencia (integración-funcional) que se define por su grado de apertura real 10.

La apertura y la funcionalización de la frontera se definen en relación a la movilidad que ella registra. La frontera como instrumento de regulación de todo Estado nacional va a oscilar de una funcionalización extrema que reduce tráfico e intercambios, a una desfuncionalización que reduce mecanismos de cóntrol y vigilancia a un simple registro estadístico. Los objetivos, la dimensión territorial que toca y la temporalidad en que se aplican, hacen de las funciones fronterizas instrumentos de política interna o internacional que inciden de manera estructural o coyuntural en estas regiones.

La función legal. Al interior de toda línea de demarcación política, existe un conjunto de normas e instituciones jurídicas que regulan la existencia de un Estado-nación. La frontera sirve para delimitar el área en la cual se aplica el 10 Teune H., Mlinarz Z., "Development and openness of systems", in Boundaries and regions, Lint Ed, Triestre, 1973. pp. 117-240; Klemencik V., "The open border and regions as new regienal geographic phenomen", Congrés International de Géographie, Budapest, 1978, pp. 145-150. 
derecho positivo de un Estado, principio de orden necesario que va a dotar de eficacia a los órganos judiciales. La función legal es, en consecuencia, la función más estable; ella permanece inclusive en el caso de la desfuncionalización total de las fronteras.

La funcion fiscal. Tradicionalmente, la función fiscal se relacionaba con la imposición que se hacía sobre los productos provenientes del extranjero. La complejidad actual de los espacios económicos internacionales tiende a desbordar esta visión para que se desarrollen mecanismos inductivos.

Geográficamente, la contigüidad, salvo en casos extremos, hace de los espacios fronterizos entidades relativamente "permeables" o "fluidas". En esta dimensión, la función fiscal es una pieza maestra en el diseño de la política económica. Ella procede a proteger ciertas industrias, formas de intercambio o selección de factores. Su selectividad puede definirse también espacialmente, como sucede con las zonas y perímetros libres en ciertos casos.

En conjunto, las medidas fiscales sirven a todo Estado para prohibir o incitar el desarrollo de actividades. Su impacto es directo o indirecto al promover inversiones directas o flujos pendulares de capitales, hombres y bienes en relación a las diferencias que ellas introducen.

Función de control. La vigilancia y el control de hombres y bienes constituye una función externa de la frontera. Ella implica normas de prohibición o de exclusión y la existencia de un aparato de ejecución. El control se ejerce en diferentes dominios.

La frontera funciona como filtro a partir de criterios cualitativos o cuantitativos del movimiento de bienes y factores. El ejemplo frecuentemente evocado son los controles sobre embalajes, etiquetajes o cuotas para ciertos productos, y los que se desarrollan sobre los movimientos migratorios legales que están sujetos a controles de raza, etnia, ideologia, salud, etc ${ }^{11}$, La definición y aplicación de controles entre Estados fronterizos tiende a reflejar la relación de fuerzas y la capacidad de negociación que posee cada uno de ellos, que en última instancia obedecen al desarrollo histórico de sus respectivas estructuras socioeconómicas.

Espacialmente, las funciones intervienen como reguladoras en una dimensión temporal del proceso de integración-desintegración territorial de los espacios fronterizos. Las funciones, al determinar directa o indirecta11 En este dominio las polfticas de migración de Estados Unidos y Australia son ejemplos en la aplicación de tales criterios. p. ej. Bustamante J., Espaldas mojadas: materia prima para la expansión del capital norteamericano, Cuadernos del CES. No. 9, México, 1975, pp. 6-13. 
mente el nivel de apertura de estos espacios, operan como barreras "selectivas" que inciden directamente en la estructuración organizacional y material (espacio construido) de estas regiones. Su originalidad es la de ser extremadamente dinámicas por depender en su formación y ejecución de objetivos políticos, los cuales obedecen frecuentemente a decisiones políticas producto de necesidades parciales (nacionales) en un contexto binacional. Por esto último, cada acto de control es percibido como un costo político, económico o a la inversa, según la posición relativa de la entidad reguladora y el bien o agente afectado en su ámbito de desplazamiento.

\section{La region fronteriza.}

La frontera en su dimensión espacial tiene un carácter dinámico, lo que reproduce a uno y otro lado de su traza las diferencias morfológicas, estructurales y funcionales que constituyen simultáneamente factores y producto de su organización espacial. La configuración territorial que resulta es, de manera directa, producto de la demarcación, y de manera indirecta, resultado de la yuxtaposición de al menos dos modos de organización institucional y del desarrollo histórico de las estructuras productivas.

A uno y otro lado de la línea fronteriza, las diferencias en los niveles de concentración económica, y de formas construidas en relación al espacio, constituyen la forma tangible en que se integran subregionalmente los componentes de la región fronteriza internacional. La presencia de la frontera estimula el desarrollo de zonas específicas de comportamiento, cuya dimensión territorial obedece menos a la decisión política que a la división internacional del trabajo 12 , que materialmente remiten a estructuras productivas subregionales (en un contexto internacional) desiguales.

La dinámica en la que se sustenta el espacio fronterizo es válida como instrumento de análisis del concepto de movilidad. La movilidad, como concepto, define de manera general un proceso de cambio, cuya connotación posee una doble dimensión espacio-temporal. Especificamente, el concepto integra localización y movimiento en base a un sistema de factores que intervienen en él.

La movilidad, al referir a un cambio ya sea de objetivos o de agentes en relación a sus similares circundantes, facilita distinguir factores y procesos de concentración y dispersión en su desarrollo histórico. Como fenómeno que obedece a la influencia de determinaciones de carácter cualitativo o cuantitativo, y debido a la delimitación física que le confieren el derecho interno e 12 Petras E., "The role of national boundaries in a cross national labour market", Urban Intemational Review, Vol. No. 4 
internacional el espacio fronterizo permite situar todo desplazamiento en relación a la línea fronteriza. La movilidad, en su dirección, intensidad y temporalidad, constituye la variable a identificar en el análisis de la etimología espacial fronteriza.

Si por necesidad analitica se conviene en identificar la movilidad interregional (intemacional) en relación a ciertas variables privilegiadas como son capital, trabajo y bienes, considerándolas agentes activos en la estructuración de redes territoriales y de organización, la región fronteriza puede, en relación a su fragmentación relativa de carácter internacional, analizarse a la luz de la especialización de cada una de sus subregiones. Estas estructuras subregionales se constituyen en factor y producto de los mecanismos de ajuste cuyas reglas de interdependencia relativamente flexibles van a dar lugar a formas territoriales particularmente inestables.

Espacialmente, tales sistemas regionales internacionales se definen en relación a la localización y al proceso de movilidad dentro del cual se integran los distintos agentes a uno y otro lado de la frontera. Los factores locacionales en la región fronteriza tienen un carácter especialmente complejo, en virtud de que se apoyan en una doble disponibilidad territorial de recursos y de jurisdicción polf́tico-administrativa que los convierte generalmente en realidades estructurales de alta coyuntura, cuya expresión es una movilidad generalizada.

Al estudiar la región fronteriza en función de sus niveles variables de apertura, de los desplazamientos de factores identificados y de estructuras territoriales, como materialidad que refleja la práctica espacial que la configura, aquella aparece como una entidad compleja factible de estudiarse en relación a su dimensión económica, demográfica y a las estructuras territoriales en cuya cohesión relativa descansa su organización.

Los factores económicos. F. Perroux, al referirse al fenómeno de transnacionalización al que eran particularmente sensibles las regiones fronterizas, lo hacía aparecer como una erosión de la soberanía nacional 13. El desarrollo de la movilidad generalizada en la mayoría de las sociedades occidentales que tienden hacia una integración mayor entre sus economfas 14 , incide cada vez más en el plano político. El desarrollo del proceso lleva de manera implícita a desvanecer, cada vez más, la concepción de autarquía al que se asimilaba todo Estado-nación y por extensión toda economía capitalista. 13 Perroux F., L'Europe sans rivages, París, 1954, cit. por Guichonnet P., Raffestin E., Géographie des frontiéres, PUF, París, 1974, pp. 153.

14 Bajo diferentes enfoques, el fenómeno es analizado particularmente por: Adyalot Ph., Dynamique spatiale et développment inégal, Economica, París 1980; Gaudemar J.P., Movilidad del trabajo y acumulación de capital., Era Ed.; México, 1979.; La mobilité genérale, Ed, Du champ urbain, Paris, 1979. 
Dentro de este contexto global, la dimensión regional de la frontera tiene la cualidad de hacer emerger las condiciones de desigualdad como fenómeno localizado. Esta condición de existencia refleja localmente el desarrollo de formas territoriales y espaciales que traducen el conjunto de discordancias existentes entre economías nacionales contiguas. Estas diferencias, al desarrollarse históricamente, dan lugar a un proceso espacio-temporal de estructuración y desestructuración por la extrema sensibilidad de estas zonas.

Como lo hemos señalado en la primera parte, históricamente la región fronteriza experimenta un desplazamiento que va de la rigidez derivada de las barreras selectivas originadas en la figura juridica, hacia formas integradas de producción entre espacios nacionales compartidos. Este desplazamiento lleva sucesivamente a representar la frontera como zona de mercado amputado y polo de desarrollo incompleto ${ }^{15}$, resultado de su presencia y del clima de desconfianza imperante. En la actualidad, la internacionalización de las economras, su expansión geográfica y la emergencia de procesos segmentados de producción técnica y locacionalmente, convierte a los espacios fronterizos en territorios estratégicos para el desarrollo global del capitalismo.

La fragilidad estructural por la cual las fronteras políticas se consideraban economías deprimidas y vulnerables, es en la actualidad una ventaja para el desarrollo de procesos productivos cuyo desarrollo tecnológico promueve la segmentación de los mercados nacionales de trabajo en base a la delocalización geográfica de las actividades.

Esta transformación cualtitativa del papel de la frontera la convierte cada vez más en una entidad regional caracterizada por su extremo dinamismo. Territorios considerados de alta coyuntura tienden a reflejar las diferencias a corto plazo provocadas por las variaciones de salarios y precios. Ambas variables particularmente interrelacionadas, dan lugar a movimientos de ajuste extremadamente complejos. Los movimientos expresan las diferencias estructurales y monetarias con base en las cuales se desarrolla la interdependencia fronteriza, de modo que los flujos de trabajo se desplazan en razón de variaciones que inciden relativamente en salarios y precios. Estas-variaciones, como catalizadores de movilidad, adquieren una dimensión temporal y territorial según sean los excedentes de trabajo y la capacidad de absorción de las respectivas estructuras productivas. Las mismas variaciones en salarios y precios-inciden en el movimiento de mercancías y consumidores que se desplazan de uno a otro territorio en respuesta a los cambios que experimentan.

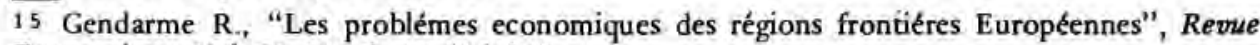
Economique, Vol. 21, No. 6, pp. 889-917. 
El carácter fluctuante de la región con respecto a sus respectivas economías nacionales, la movilización general en que se desarrolla el proceso productivo, las ventajas comparativas que de ella se desprenden y la fluidez del capital regulada por políticas parciales y bilaterales, convierten a estas regiones en los espacios privilegiados para la expansión económica.

Los factores demográficos. Geográficamente, las poblaciones fronterizas son marginales, frecuentemente también por razones lingürsticas o étnicas. En la práctica, esta situación marginal refleja la doble participación de estas poblaciones en sistemas relativamente diferentes.

Espacios dinámicos por responder a un proceso de integración-desintegración en su doble dimensión interna-internacional, el factor poblacional aseguró en el pasado la explotación de nuevos recursos naturales para integrarse en la actualidad a nuevas formas de organización. La población fronteriza es particularmente hetereogénea en relación a las regiones interiores respecto a las cuales se subraya su carácter dinámico. Al estar inmersa en una ósmosis cuyo dinamismo es catalizado por su misma localización, hace que su dinámica, estructura y distribución reflejen la doble dimensión en la que se desarrolla.

La región fronteriza suele experimentar procesos migratorios intensos que en relación a la línea fronteriza pueden situarse a varios niveles: migraciones internas para las cuales el límite es la propia frontera; migraciones alternantes (commuters), cuya dinámica es resultado de las condiciones estructurales y coyunturales de interdependencia; $y$, por último, las migraciones internacionales, que convierten estos espacios marginales en puertas de salida o entrada 16 .

Demográficamente, las regiones fronterizas son los espacios de disonancia de los desequilibrios estructurales y coyunturales de cada territorio nacional. Los movimientos interregionales e internacionales que vehiculan en ellos operan como procesos de ajuste a corto y mediano plazo merced a las fluctuaciones políticas y económicas a que las someten las disposiciones formales y de las organizaciones económicas. La dirección de flujos y sus intensidades explican concentración y distribución de la población de las regiones binacionales en relación a la línea de demarcación internacional. La frontera, al funcionar como filtro de estos movimientos de ajuste entre los excedentes y las demandas interregionales e internacionales de trabajo, define la dimensión, localización y presiones que los desplazamientos ejercen sobre sus respectivos espacios construidos.

16 $\mathrm{La}$ frontera tiene de esta manera un triple papel con respecto al movimiento demográfico: es etapa, base y refugio para los migrantes. Revel Mouroz J., Bataillon C, "Les migrations mexicaines vers la frontiere nord du Mexique", Tiers Monde, Vol. No. pp. 73-75. 
Espacio fronterizo y estructura espacial. La estructura espacial como representación física de la organización social asegura su continuidad en base a una cohesión relativa. En el contexto del desarrollo capitalista, la frontera es un espacio límite del conflicto que opone la unicidad territorial del Estado y el desplazamiento transnacional que genera las nuevas formas de organización económica.

Las interacciones que se desarrollan tienden a dar forma a una estructura espacial que sigue ciertas formas generales y específicas que traducen la intensidad y dirección en que se desarrolla históricamente cada espacio. En estas condiciones, las "esferas de influencia" como dimensión espacial se desarrollan en base a la presencia de la frontera polftica y al sesgo que ella introduce en la organización del espacio. Los centros y espacios circundantes no constituyen formas concéntricas como lo define el análisis espacial tradicional-son, por el contrario, formas materiales cuya estructura refleja el carácter desigual que en el tiempo, poseen las estructuras espaciales de talla nacional. De esta manera, la frontera ejerce una influencia directa $\mathrm{e}$ indirecta en la configuración de redes, tamaño de campos y su forma.

a) Tamaño y forma. Si se supone que los movimientos pueden fluir libremente en cualquier dirección, se tiene que admitir que en su mayoría todo desplazamiento está restringido a alguna suerte de canal. En consecuencia, rutas y redes territoriales son factores materiales que dan coherencia a la organización espacial.

Teóricamente, toda ruta se desarrolla según la ley del mínimo esfuerzo "lex parsimoniae", Esta noción que remite originalmente a una dimensión de carácter espacio-temporal tiene su traducción económica en términos de costos: costo en relación a la distancia, al tiempo o a una combinación de ambos en términos de transportabilidad.

Así, toda red obedece a los objetivos definidos por cada uno de los agentes activos en la toma de decisión, como son los objetivos políticos y los de carácter económico. Además, de una manera general, toda red se desarrolla según criterios de distancia mínima (individual), de optimalidad (en el conjunto) y factores de distorsión, los cuales intervienen en su configuración.

Territorialmente, el tamaño y la forma de los campos a los que da lugar la presencia de la frontera, están dados por los diferentes criterios de continuidad que intervienen en su configuración. En primera instancia, cada Estado tiende a asegurar su continuidad política de una manera absoluta en el seno de su territorio, mientras que de otra parte, las entidades económicas plantean su continuidad siguiendo criterios de optimización que van 
más allá de la división territorial de carácter nacional. En estas condiciones, los espacios económicos integran el conjunto de diferenciales a que da lugar la presencia de la frontera, respondiendo a sus objetivos internos de optimización.

El tamaño de las superficies de integración varía según sean los niveles de apertura (en función de las barreras selectivas) de cada frontera internacional, del desarrollo del transporte (producto del desarrollo tecnológico) y de los niveles de integración territorial que existan entre redes nacionales. En estas condiciones, su fluidez tiene una dimensión temporal; en el corto plazo, según las diferencias específicas que experimenten los sujetos y los bienes en desplazamiento; en el mediano y largo plazo, por los procesos de integración socioeconómica y el desarrollo del transporte que relativiza la distancia. 17 Las transformaciones a que están sujetos los espacios fronterizos introduce cambios en su configuración general en relación a los factores de localización y al desarrollo de una gama cada vez mayor de interacciones a que da lugar la tendencia generalizada a la apertura.

La distribución territorial desigual que caracteriza la mayor parte de los espacios fronterizos es la forma material que da fe de las transformaciones históricas en las que se desarrolla cada espacio nacional. Esta originalidad localizada lleva a plantearlo en relación a las formas teóricas que en base a la forma concéntrica, tienden a identificar el espacio fronterizo como una forma truncada o distorsionada según el carácter dinámico de que se le dote.

En el primer caso, la forma truncada remite al estudio clásico de A. Losch 18 situado sobre la frontera México-EE.UU. Siguiendo los criterios de interferencia tanto de orden físico como político, este autor señala la presencia de la frontera como una forma que modifica la forma real del campo. Esta variación central remite a la formulación de su esquema de espacio truncado (Fig. 1). En el primer caso (Fig. 1.a.) se plantea la posibilidad de una fluidez total en todas sus partes; en el segundo caso (Fig. 1.b.), plantea el pasaje como limitado a un puesto de aduana B. Finalmente, la figura 1.c. hace emerger la presencia de una barrera neutral (río) con un único punto de pasaje $\mathbf{B}$.

La forma de los campos distorsionados obedece a transformaciones mayores que los de movimientos relativamente locales. El desarrollo de fenómenos de excentricidad, como resultado de los efectos asimétricos, muestra la emergencia de la frontera en relación a las condiciones estructurales entre las econom Ias nacionales y regionales. En base a un esquema que conjugue simul-

17 Johnston R.J. Spatial structures, Metahuen and Co., London, 1977, p.7

18 Losch A., The economics of location, John Wiley and Sons, N.Y. 1967, p. 196. 
FIGURA 1. Campos trunçados por fronteras políticas y naturales

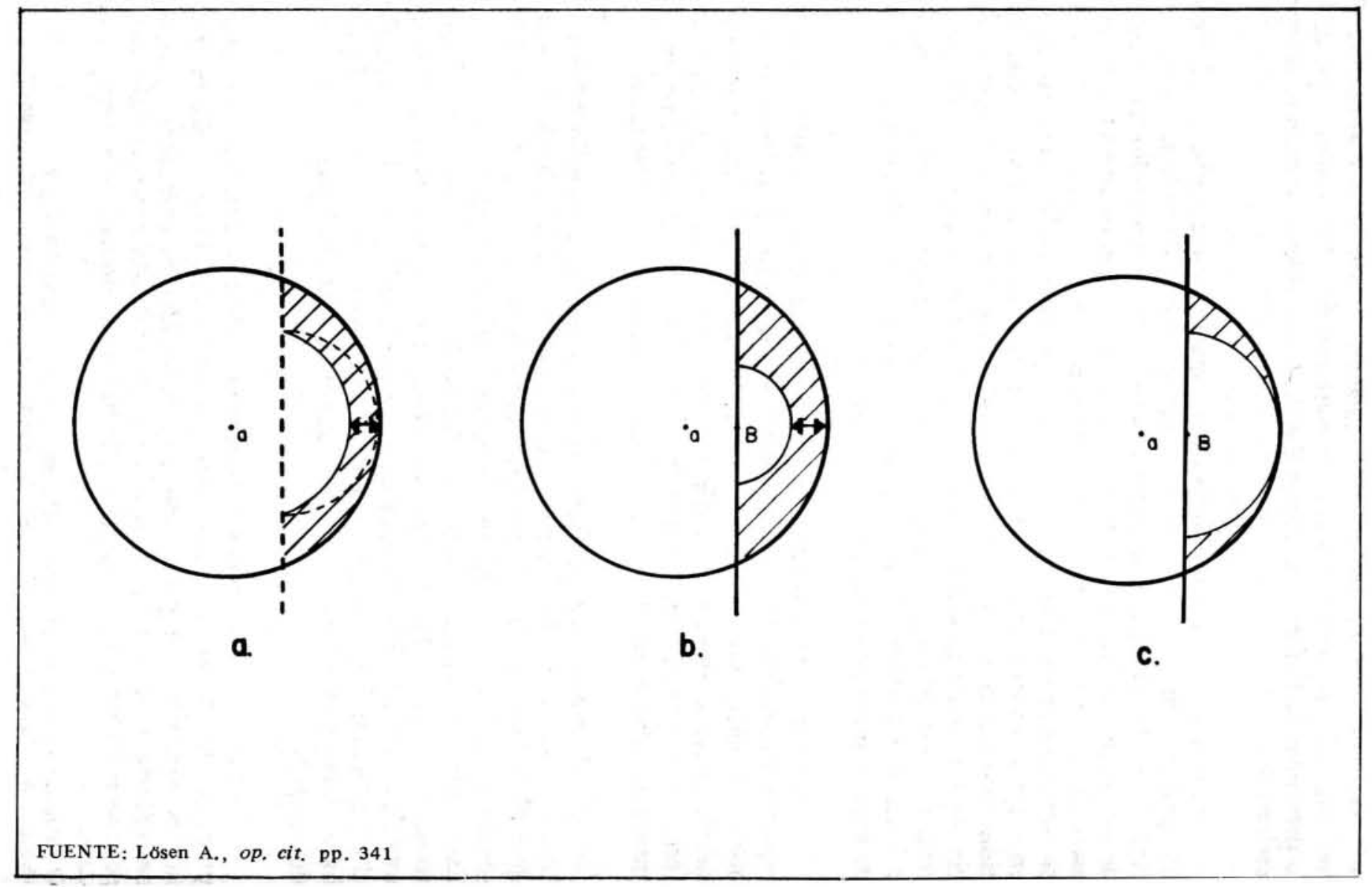


táneamente la potencialidad y los efectos de polarización que ejerce la frontera en el ámbito político, económico y geográfico, dicha relación diferencial podría tentativamente explicarse en base a un diagrama.

En la figura 2, el fenómeno es descrito en un ámbito local y nacional. El efecto de estructura dominante se ejerce del país I al II. En el primer caso, de carácter local $(2, a$,$) , los efectos tangibles son: un movimiento en los dos sen-$ tidos de mercancías (X-M), que no es privativo de esta escala pero se cataliza; un flujo definido de capitales de I a II, que por su ubicuidad tiene un carácter global, y un flujo de trabajo a través de migraciones temporales y alternantes (commuters) vehiculando de II a I por la diferencia estructural que se supone dada en ese sentido (reflejada en términos de empleo y salario). En su dimensión nacional (2.5), el proceso de polarización opera por la importación de capitales provenientes de la empresa transnacional (en nuestro ejemplo) sobre el territorio fronterizo del país II, mientras que en el sentido inverso, la migración interna del pars I adquiere una dimensión internacional al desplazarse hacia el territorio fronterizo del país II. La frontera, al convertirse en filtro por sus funciones de regulación de tales desplazamientos, se convierte en factor de localización y producto territorial de la desigualdad imperante 19.

El desarrollo fronterizo es expresión del desarrollo histórico y de las diferentes formas en que interaccionan dos entidades nacionales. Espacialmente, el proceso de desigualdad en que se estructuran las relaciones se expresa en intensidad y extension, que puede rebasar la sola dimensión regional.

b) Región y ciudad fronteriza. Dentro del corte complementario a que se remite generalmente el espacio como regional-urbano, el espacio fronterizo es susceptible de asimilarse regionalmente como espacios agrícolas y espacios - urbanos. Esta simplificación permite analizar algunas tendencias de carácter original que se desarrollan en cada una de estas dimensiones.

En la transformación del espacio agrícola, la presencia de la frontera es un factor diferencial que va a favorecer el desarrollo de la especulación por d suelo. El primer mecanismo de este tipo tiene que ver con las demandas de suelo agrícola y con el desarrollo de una especulación inmobiliaria de zonas secundarias o de carácter residencial.

\footnotetext{
19 La movilidad como producto de la desigualdad es el factor de transformación espacial. En ote sentido, la dimensión del movimiento se modifica pero el mecanismo subsiste. A manera de ezemplo, la movilidad entre Francia y Alemania siempre ha existido, pero su dimension es rgional. Gendarme op. cit., pp. 903-904, Urban S, "L'intégration économique européene er l'évolution regionale de part et d'autre du Rhin (Alsace, Baden Bâle)", Revue Economique, Fol 21, No. 6, pp. 603-633.
} 

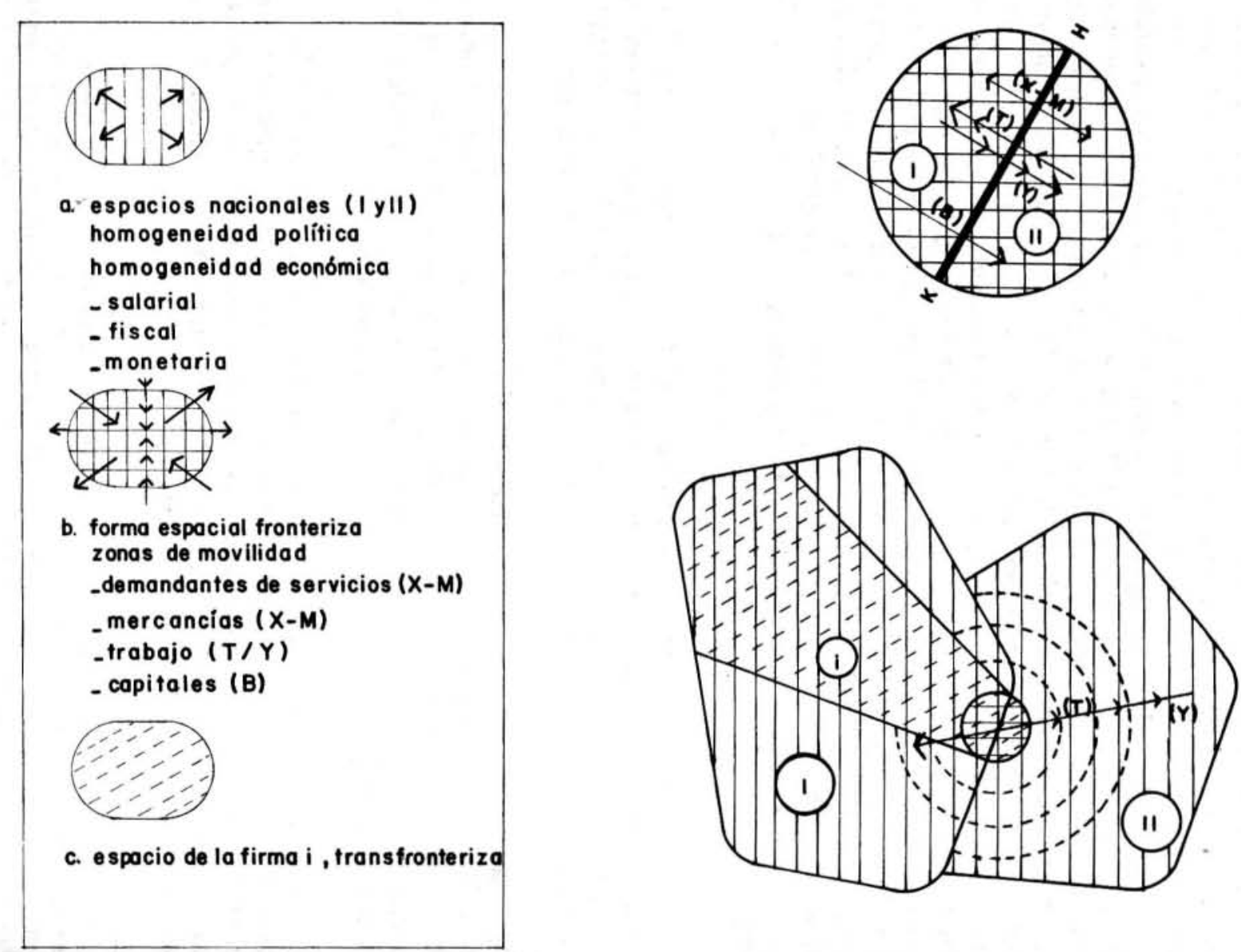
La demanda de suelos agrícolas puede tener efectos múltiples, pero en general es la consecuencia de una rarefacción de las tierras agrícolas en la economía más dinámica. Las diferencias substanciales en los precios, que impera entre los dos países, o la simple necesidad de extender las superficies de explotación, van a favorecer el fenómeno. Frente a las limitaciones de carácter legal que impiden frecuentemente la propiedad de extranjeros en zonas fronterizas, siempre existe la posibilidad de rentar tierras de explotación o financiar directamente a agricultores, acciones que en conjunto van a favorecer las tendencias de alta coyuntura en estos territorios.

El desarrollo de una especulación inmobiliaria destinada principalmente a residencias secundarias se apoya en la existencia de condiciones ambientales favorables y de diferencias monetarias sensibles que favorezcan el desplazamiento de capitales. Este fenómeno de carácter acumulativo tiende a subrayar los efectos asimétricos de carácter sociogeográfico como son la transformación del medịo ambiente y la modificación substancial de las funciones económicas.

Es evidente que los Estados pueden hacer frente a esas apropiaciones que crean desequilibrios en sus confines. En estos casos, el Estado sobre el que se desarrolla el proceso de expansión va a instaurar medidas de regulación con el objeto de reducir las influencias nocivas que modifican sus estructuras territoriales y funcionales.

De una manera general, las ciudades fronterizas tienden a reflejar el carácter dinámico a que está expuesta la frontera. Las ciudades son los espacios privilegiados por los movimientos interregionales e internacionales que son la expresión de las desigualdades estructurales. Como expresión territorial de la concentración económica y demográfica, los centros urbanos fronterizos son la manifestación material de las condiciones estructurales y coyunturales en las que se organizan las relaciones globales y locales de carácter binacional.

Como espacio construido, y debido a su localización regional, las ciudades fronterizas van a operar como filtros (relativos a sus funciones y a su grado de apertura) de las diferentes formas de movilidad. Esto las lleva a que, territorialmente (espacio construido), las ciudades amplifiquen las condiciones favorables y desfavorables en que se desarrollan los procesos de expansión y/o integración.

En estas condiciones, la inestabilidad de estos centros urbanos es una forma de materializar la función simultánea a la que está expuesto su desarollo: como espacio de equilibrio a la movilidad interna, y como espacio 
de desequilibrio frente a la incompatibilidad internacional entre flujos y mecanismos de regulación que resultan de la desigualdad entre países.

\section{CONCLUSION}

La frontera no es solamente un instrumento de demarcación territorial como lo sugiere su definición formal; no es una entidad neutra, ni un instrumento ausoluto de cada Estado según las líneas discretas y frágiles a que las relegan las cartas geográficas.

Lo relevante es que la frontera es un fenómeno social. Como tal, da lugar a la presencia de conflictos, discordancias y transformaciones que la ubican en una dimensión dinámica. Esto la lleva a engendrar costos, deseconomías y desfuncionalizaciones cuyos efectos inciden directamente sobre las poblaciones fronterizas e indirectamente sobre los complejos nacionales.

El origen del fenómeno remite a una diferencia estructural que traduce el desarrollo histórico de cada uno de los espacios nacionales involucrados, La organización de estos espacios aparece como un subproducto del efecto de dominación con que se vinculan estas regiones; las relaciones asimétricas a que da lugar su emergencia van a marcar las relaciones que se llevan a cabo entre poderes interestatales, estructuras socioeconómicas y sus diferentes formas de movilidad.

Regionalmente, el análisis diferencial facilita la identificación de transformaciones en las cuales la complejidad espacio-temporal se expresa por el desarrollo de las contradicciones entre las organizaciones sociales y los espacios construidos. El espacio fronterizo, como complejo social, posee la originalidad de establecer un punto fijo en el análisis de formas históricas que resultan de la experiencia, percepción y construcción del espacio. 